Article

\title{
Simple New Proofs of the Characteristic Functions of the $F$ and Skew-Normal Distributions
}

\author{
Jun Zhao ${ }^{1}$, Sung-Bum Kim ${ }^{2}$, Seog-Jin Kim ${ }^{3}$ and Hyoung-Moon Kim ${ }^{2, *}$ (i) \\ 1 School of Mathematics and Statistics, Ningbo University, Ningbo 315000, China; zhaojun1@nbu.edu.cn \\ 2 Department of Applied Statistics, Konkuk University, Seoul 05029, Korea; kimsb0707@hanmail.net \\ 3 Department of Mathematics Education, Konkuk University, Seoul 05029, Korea; skim12@konkuk.ac.kr \\ * Correspondence: hmkim@konkuk.ac.kr
}

Received: 23 November 2020; Accepted: 9 December 2020; Published: 10 December 2020

\begin{abstract}
For a statistical distribution, the characteristic function $(\mathrm{CF})$ is crucial because of the one-to-one correspondence between a distribution function and its $\mathrm{CF}$ and other properties. In order to avoid the calculation of contour integrals, the CFs of two popular distributions, the $F$ and the skew-normal distributions, are derived by solving two ordinary differential equations (ODEs). The results suggest that the approach of deriving CFs by the ODEs is effective for asymmetric distributions. A much simpler approach is proposed to derive the $C F$ of the multivariate $F$ distribution in terms of a stochastic representation without using contour integration. For a special case of the multivariate $F$ distribution where the variable dimension is one, its $C F$ is consistent with that of the former (univariate) $F$ distribution. This further confirms that the derivations are reasonable. The derivation is quite simple, and is suitable for presentation in statistics theory courses.
\end{abstract}

Keywords: characteristic function; ordinary differential equation; $F$ distribution; skew-normal distribution; asymmetric distribution

\section{Introduction}

For any distribution function, its characteristic function (CF) always exists because of the integrability for every bounded and measurable function with respect to any distribution over the real line. The CF plays an important role in statistics due to the one-to-one correspondence between a distribution function and its CF. By the continuity theorem, the one-to-one correspondence relationship is also continuous. According to [1,2], CFs have many pleasant properties, e.g., every characteristic function is uniformly continuous on the whole real line. Owing to the properties of the $C F$, the calculation of the $C F$ for each distribution has received much interest in statistical literature.

For example, the $F$ distribution (also known as Snedecor's $F$ distribution or the Fisher-Snedecor distribution), which was derived in the year 1924 in [3], has been frequently used in many studies, particularly as a null distribution of test statistics. Its CF has been derived in many studies, such as [4-7], but the CF of the F distribution was listed incorrectly. The author of [8] corrected this using contour integration as

$$
\psi_{X}(t)=E e^{i t X}=\frac{\Gamma\left(\frac{v_{1}+v_{2}}{2}\right)}{\Gamma\left(\frac{v_{2}}{2}\right)} U\left(\frac{v_{1}}{2}, 1-\frac{v_{2}}{2},-\frac{v_{2}}{v_{1}} i t\right), \quad t \in \mathbb{R}, i=\sqrt{-1},
$$


where the random variable $X>0$ follows the $F$ distribution with degrees of freedom $v_{1}>0$ and $v_{2}>0$, and $U(\cdot)$ is Tricomi's (confluent hypergeometric) function proposed in [9] with its specific expression in Appendix A. The application of this CF can be found in many studies. For instance, ref. [10] utilized this $\mathrm{CF}$ to evaluate the distribution of a linear combination of independent $F$ random variables, which can be applied to compute the exact confidence intervals for the common mean of several normal populations. In [11] the authors applied this CF to obtain the distribution of an average $F$-statistic for testing linear asset pricing models. In [12], this CF is applied to calculate the moment of the ratio between Euclidean distances in high dimensional spaces.

Another example is regarding a skew-normal distribution that was first introduced in [13]. The skew-normal distribution generalizes the normal distribution to allow for non-zero skewness. For instance, it is applied to measure various body levels for sports athletes in [14], to measure prices of bottles of Barolo wine in [15], and to measure different chemical measurements of Piedmont wines in [16]. For a random variable $Y$ with values in $\mathbb{R}$ following the skew-normal distribution with the skewness (or slant) parameter $\lambda$, that is, $Y \sim S N(0,1, \lambda)$, the CF given by [17-19] and [15] is

$$
\psi_{Y}(t)=E e^{i t Y}=e^{-\frac{t^{2}}{2}}\left(1+\sqrt{\frac{2}{\pi}} i \int_{-\infty}^{\delta t} e^{\frac{v^{2}}{2}} d v\right), t \in \mathbb{R},
$$

with $\delta=\frac{\lambda}{\sqrt{1+\lambda^{2}}}$. In [19] the authors also obtained different forms of the CF of the skew-normal distribution using contour integration. This CF is employed to estimate the location and scale parameters as a special case of the skew-symmetric distribution in [20]. Taking this CF as a special case, the goodness-of-fit test is done based on CFs in [21].

Because statistics students are unfamiliar with contour integration, the rigorous derivation of CFs based on such integration is mostly avoided. Using ordinary differential equations (ODEs), ref. [22] presented a proof of the CF of a Student's $t$-distribution. The current study extends the ODE method to derive the above two CFs, which are for asymmetric distributions.

The further objective of this study is to derive the CFs of some multivariate distributions without contour integration. For the multivariate $F$ distribution introduced in $[23,24]$ proposed its $C F$ through the transformation of the general inverted Dirichlet distribution, and the calculation is done via the contour integrals. In terms of a simple stochastic representation, the derivation of the CF of the multivariate $F$ distribution proposed in this study is much simpler than the one employing the contour integrals.

In Section 2, by applying the ODEs, we first calculate the CF of the $F$ distribution. The reason that this CF consists of a Tricomi's function can be explained by the computing process. The calculation for the ODEs of the skew-normal distribution follows. The Faddeeva function is involved in the derivation of the CF for the skew-normal distribution. Even though it is not always easy to obtain the solution for an ODE, which can further yield the $\mathrm{CF}$, solution for the ODE of the $F$ distribution is found in Kummer's equation (see Appendix A) and that for the ODE of the skew-normal distributions is found in a differential equation of the Faddeeva function (see Appendix B). In Section 3, the CF of the multivariate $F$ distribution is also derived using simple stochastic representation without using contour integration. Finally, a short conclusion is provided in Section 4. 


\section{Deriving the CFs by ODEs}

\subsection{Derivation for the F Distribution}

For the random variable $X \sim F\left(v_{1}, v_{2}\right)$, the probability density function (PDF) is

$$
f_{X}(x)=\frac{\Gamma\left(\frac{v_{1}+v_{2}}{2}\right)}{\Gamma\left(\frac{v_{1}}{2}\right) \Gamma\left(\frac{v_{2}}{2}\right)}\left(\frac{v_{1}}{v_{2}}\right)^{\frac{v_{1}}{2}} x^{\frac{v_{1}}{2}-1}\left(1+\frac{v_{1}}{v_{2}} x\right)^{-\frac{v_{1}+v_{2}}{2}}, x>0,
$$

with degrees of freedom $v_{1}>0$ and $v_{2}>0$, where $\Gamma()$ is a Gamma function.

It is first proved for $v_{2}>4$ and then $v_{2}>2$. Finally, the result will be proved for $v_{2}>0$. The first derivative at $x$ of $f_{X}(x)$ leads to

$$
2\left(v_{2}+v_{1} x\right) x f_{X}^{\prime}(x)+v_{1}\left(v_{2}+2\right) x f_{X}(x)-v_{2}\left(v_{1}-2\right) f_{X}(x)=0 .
$$

When $v_{2}>4$, an integration by parts to this differential equation further results in

$$
\begin{aligned}
0 & =\int_{0}^{\infty}\left[2\left(v_{2}+v_{1} x\right) x f_{X}^{\prime}(x)+v_{1}\left(v_{2}+2\right) x f_{X}(x)-v_{2}\left(v_{1}-2\right) f_{X}(x)\right] e^{i t x} d x \\
& =-v_{1} v_{2} E\left(e^{i t X}\right)+\left(v_{1} v_{2}-2 v_{1}-2 i t v_{2}\right) E\left(X e^{i t X}\right)-2 i t v_{1} E\left(X^{2} e^{i t X}\right)
\end{aligned}
$$

where all integrals exist because $v_{2}>4$. Combining with the properties of the $C F$,

$$
\psi_{X}^{\prime}(t)=i E\left(X e^{i t X}\right) \text { and } \psi_{X}^{\prime \prime}(t)=-E\left(X^{2} e^{i t X}\right),
$$

the ODE of the $F$ distribution for the CF $\psi_{X}(t)$ with $v_{2}>4$ is

$$
2 v_{1} t \psi_{X}^{\prime \prime}(t)+\left[2 i v_{2} t-v_{1}\left(v_{2}-2\right)\right] \psi_{X}^{\prime}(t)+i v_{1} v_{2} \psi_{X}(t)=0 .
$$

Referring to Appendix A, if $w(z)$ satisfies Kummer's Equation (A1), that is,

$$
\operatorname{ing} z w^{\prime \prime}(z)+(b-z) w^{\prime}(z)-a w(z)=0,
$$

then $-\frac{v_{2}}{v_{1}} i z w^{\prime \prime}\left(-\frac{v_{2}}{v_{1}} i z\right)+\left(1-\frac{v_{2}}{2}+\frac{v_{2}}{v_{1}} i z\right) w^{\prime}\left(-\frac{v_{2}}{v_{1}} i z\right)-\frac{v_{1}}{2} w\left(-\frac{v_{2}}{v_{1}} i z\right)=0$ follows. Furthermore, $g_{*}(z)=$ $w\left(-\frac{v_{2}}{v_{1}} i z\right)$ satisfies $2 v_{1} z g_{*}^{\prime \prime}(z)+\left[2 i v_{2} z-v_{1}\left(v_{2}-2\right)\right] g_{*}^{\prime}(z)+i v_{1} v_{2} g_{*}(z)=0$. Therefore, the complete solution for (4) is

$$
\psi_{X}(t)=C_{1} M\left(\frac{\nu_{1}}{2}, 1-\frac{v_{2}}{2},-\frac{v_{2}}{v_{1}} i t\right)+C_{2} U\left(\frac{\nu_{1}}{2}, 1-\frac{v_{2}}{2},-\frac{v_{2}}{v_{1}} i t\right),
$$

where $C_{1}$ and $C_{2}$ are arbitrary constants except that $M(a, b, z)$ (Kummer's function of the first kind) does not exist when $b$ is a nonpositive integer. $U(a, b, z)$ is Tricomi's function with specific expression given in Appendix A. 
The standard properties of a CF $\psi_{X}(t)$, which include $\lim _{t \rightarrow 0} \psi_{X}(t)=1$ and $\left|\psi_{X}(t)\right| \leq 1$ for all $t \in \mathbb{R}$, can be combined with (A2)-(A5) in Appendix A, that is, with

$$
\begin{aligned}
& \lim _{|t| \rightarrow 0} \psi_{X}(t)=C_{1}+C_{2} \frac{\Gamma\left(\frac{v_{2}}{2}\right)}{\Gamma\left(\frac{v_{1}+v_{2}}{2}\right)}+\mathcal{O}(z), \text { as } z \rightarrow 0, \\
& \lim _{|t| \rightarrow \infty} \psi_{X}(t) \sim C_{1} \frac{\Gamma\left(1-\frac{v_{2}}{2}\right)}{\Gamma\left(\frac{v_{1}}{2}\right)} e^{z} z^{\frac{v_{1}+v_{2}}{2}-1}+C_{2} z^{-\frac{v_{1}}{2}}, \text { as } z \rightarrow \infty,
\end{aligned}
$$

where $z=-\frac{v_{2}}{v_{1}} i t$, in order to conclude that the values of the constant coefficients are given by $C_{1}=0$ and $C_{2}=\frac{\Gamma\left(\frac{v_{1}+v_{2}}{2}\right)}{\Gamma\left(\frac{v_{2}}{2}\right)}$. Here, $|\operatorname{ph}(z)|=\frac{1}{2} \pi$ for all complex $z$ since the real part of $z$ is zero. $|\operatorname{ph}(z)|<\frac{3}{2} \pi-\delta$ follows for many small values of positive constant $\delta$. Consequently, the CF of the $F$ distribution with $v_{2}>4$ is given by (1).

Suppose $v_{2}>2$, and let $S \sim F\left(v_{1}, v_{2}+2\right)$ with the CF $\psi_{S}(t)$. On using the standard formula $\Gamma(v+1)=v \Gamma(v)$,

$$
\begin{aligned}
\psi_{S}^{\prime}\left(\frac{v_{2}}{v_{2}+2} t\right) & =i \frac{v_{2}}{v_{2}+2} E\left(S e^{i \frac{v_{2}}{v_{2}+2} t S}\right) \\
& =i \frac{\left(v_{1}+v_{2}\right)}{v_{2}} \int_{0}^{\infty} x e^{i t x} f_{X}(x) \frac{v_{2}}{v_{2}+v_{1} x} d x \\
& =i \psi_{X}(t)+\frac{2}{v_{1}} i t \psi_{X}^{\prime}(t),
\end{aligned}
$$

where $X \sim F\left(v_{1}, v_{2}\right)$. Furthermore, suppose that $v_{2}>0$ and $v_{2}^{*}=v_{2}+2>2$, and let $S^{*} \sim F\left(v_{1}, v_{2}^{*}+2\right)$. Through a similar argument as for $S$, the CF of $S^{*}$ can be obtained as

$$
\psi_{S^{*}}^{\prime}\left(\frac{v_{2}^{*}}{v_{2}^{*}+2} t\right)=i \psi_{S}(t)+\frac{2}{v_{1}} i t \psi_{S}^{\prime}(t)
$$

By applying the differential property of the Tricomi's function $U(a, b, z)(\mathrm{A} 6)$ to $\psi_{S}^{\prime}\left(\frac{v_{2}}{v_{2}+2} t\right)$ and $\psi_{S^{*}}^{\prime}\left(\frac{v_{2}^{*}}{v_{2}^{*}+2} t\right)$ and by using the recurrence relationship (A7), the CF of the $F$ distribution for $v_{2}>0$ is obtained as in (1).

\subsection{Derivation for the Skew-Normal Distribution}

The random variable $Y$ following a skew-normal distribution $S N(0,1, \lambda)$ has the PDF given by

$$
f_{Y}(y)=2 \phi(y) \Phi(\lambda y), \quad y \in \mathbb{R}, \lambda \in \mathbb{R}
$$

where $\phi(\cdot)$ and $\Phi(\cdot)$ are the density and distribution functions of the standard normal distribution, respectively.

When combined with the property of a density function in the standard normal distribution $\phi^{\prime}(y)=$ $-y \phi(y)$, the first derivative at $y$ of $f_{Y}(y)$ yields the following equation:

$$
f_{Y}^{\prime}(y)+y f_{Y}(y)-2 \lambda \phi(y) \phi(\lambda y)=0 .
$$


Using integration by parts and the properties of the CF in (3) again and

$$
\int_{-\infty}^{\infty} e^{i t y} \phi(y) \phi(\lambda y) d y=\frac{1}{\sqrt{2 \pi\left(1+\lambda^{2}\right)}} \psi_{V}\left(\frac{t}{\sqrt{1+\lambda^{2}}}\right)
$$

the ODE of the skew-normal random variable $Y$ for the $\mathrm{CF} \psi_{Y}(t)$ is

$$
\psi_{Y}^{\prime}(t)+t \psi_{Y}(t)-\sqrt{\frac{2}{\pi}} \delta i \psi_{V}\left(\frac{t}{\sqrt{1+\lambda^{2}}}\right)=0,
$$

where $\delta=\frac{\lambda}{\sqrt{1+\lambda^{2}}}, V$ is a standard normal random variable and $\psi_{V}\left(\frac{t}{\sqrt{1+\lambda^{2}}}\right)=e^{-\frac{t^{2}}{2\left(1+\lambda^{2}\right)}}$.

Referring to Appendix B, if $w(z)$ satisfies a differential equation of the Faddeeva function (A10), then $h_{*}(z)=e^{-\frac{z^{2}}{2\left(1+\lambda^{2}\right)}} w\left(\frac{\delta z}{\sqrt{2}}\right)$ satisfies

$$
h_{*}^{\prime}(z)+z h_{*}(z)-\sqrt{\frac{2}{\pi}} \delta i \psi_{V}\left(\frac{z}{\sqrt{1+\lambda^{2}}}\right)=0 .
$$

By Lemma A1 in Appendix B, the particular solution $w\left(\frac{\delta z}{\sqrt{2}}\right)$ is

$$
\begin{aligned}
w\left(\frac{\delta z}{\sqrt{2}}\right) & =e^{-\frac{\delta^{2} z^{2}}{2}}\left(1+\frac{2}{\sqrt{\pi}} i \int_{0}^{\frac{\delta z}{\sqrt{2}}} e^{u^{2}} d u\right) \\
& =e^{-\frac{\delta^{2} z^{2}}{2}}\left(1+\sqrt{\frac{2}{\pi}} i \int_{0}^{\delta z} e^{\frac{v^{2}}{2}} d v\right)
\end{aligned}
$$

Hence

$$
h_{*}(z)=e^{-\frac{z^{2}}{2}}\left(1+\sqrt{\frac{2}{\pi}} i \int_{0}^{\delta z} e^{\frac{v^{2}}{2}} d v\right) .
$$

Therefore, the CF of the skew-normal variable $Y$ is obtained as (2) since $\psi_{Y}(t)=h_{*}(t)$. For the CF of the multivariate skew-normal distribution introduced by [25], we refer to [19].

\section{Deriving the $C F$ of the Multivariate $F$ Distribution}

Based on the stochastic representation (5), we derive the CF of the multivariate $F$ distribution in a simple manner without resorting to contour integration.

Let $\chi_{j}, j=0,1, \cdots, p$ be an independent chi-squared variable with $v_{j}$ degrees of freedom, then

$$
X_{j}=\frac{\chi_{j} / v_{j}}{\chi_{0} / v_{0}}
$$

follows an $F$ distribution with degrees of freedom $v_{j}$ and $v_{0}$. The joint density of $\mathbf{X}=\left(X_{1}, \cdots, X_{p}\right)^{\top}$, is given by

$$
f_{\mathbf{X}}(\mathbf{x})=\frac{\Gamma(v / 2) \prod_{j=1}^{p}\left(\frac{v_{j}}{v_{0}}\right)^{v_{j} / 2}}{\prod_{j=0}^{p} \Gamma\left(v_{j} / 2\right)} \frac{\prod_{j=1}^{p} x_{j}^{v_{j} / 2-1}}{\left(1+\sum_{j=1}^{p} \frac{v_{j}}{v_{0}} x_{j}\right)^{v / 2}}, x_{j}>0, j=1, \cdots, p,
$$


with $v=\sum_{j=0}^{p} v_{j}$. This is known as the multivariate $F$ distribution and is denoted as $\mathbf{X} \sim F\left(v_{1}, \cdots, v_{p}, v_{0}\right)$ ([23]). When $p=1$, this density reduces to that of (univariate) $F$ distribution, $F\left(v_{1}, v_{0}\right)$.

Denote the $C F$ of the multivariate $F$ random vector $\mathbf{X}$ as $\psi_{\mathbf{X}}(\mathbf{t})=E\left(e^{i \mathbf{t}^{\top} \mathbf{X}}\right)=E\left[\exp \left(i \sum_{j=1}^{p} t_{j} \frac{\chi_{j} / v_{j}}{\chi_{0} / v_{0}}\right)\right]$ with $\mathbf{t}=\left(t_{1}, t_{2}, \ldots, t_{p}\right)^{\top}$. The law of iterated expectation and the independence among $\chi_{j}$ s lead to

$$
\psi_{\mathbf{X}}(\mathbf{t})=E_{\chi_{0}}\left[E\left(\prod_{j=1}^{p} \exp \left(i \frac{v_{0} t_{j}}{v_{j} \chi_{0}} \chi_{j}\right) \mid \chi_{0}\right)\right]=E_{\chi_{0}} \prod_{j=1}^{p} \psi_{\chi_{j}}\left(\frac{v_{0} t_{j}}{v_{j} \chi_{0}}\right)
$$

where $\psi_{\chi_{j}}\left(\frac{v_{0} t_{j}}{v_{j} \chi_{0}}\right)=\left(1-2 i \frac{v_{0} t_{j}}{v_{j} \chi_{0}}\right)^{-v_{j} / 2}$ and $\psi_{\chi_{j}}(t)$ is the CF of the chi-squared variable $\chi_{j}$. By the definition of the moment for the chi-squared random variable $\chi_{0}, \psi_{\mathbf{X}}(\mathbf{t})$ changes to

$$
\begin{aligned}
\psi_{\mathbf{X}}(\mathbf{t}) & =\int_{0}^{\infty} \frac{1}{\Gamma\left(v_{0} / 2\right) 2^{v_{0} / 2}} \chi_{0}^{v_{0} / 2-1} e^{-\chi_{0} / 2} \prod_{j=1}^{p}\left(1-2 i \frac{v_{0} t_{j}}{v_{j} \chi_{0}}\right)^{-v_{j} / 2} d \chi_{0} \\
& =\frac{1}{\Gamma\left(v_{0} / 2\right)} \int_{0}^{\infty} e^{-\frac{\chi_{0}}{2}}\left(\frac{\chi_{0}}{2}\right)^{\sum_{j=0}^{p} v_{j} / 2} \frac{1}{\chi_{0}} \prod_{j=1}^{p}\left(\frac{\chi_{0}}{2}-\frac{v_{0}}{v_{j}} i t_{j}\right)^{-v_{j} / 2} d \chi_{0} \\
& =\frac{1}{\Gamma\left(v_{0} / 2\right)} \int_{0}^{\infty} e^{-y} y^{\sum_{j=0}^{p} v_{j} / 2-1} \prod_{j=1}^{p}\left(y-\frac{v_{0}}{v_{j}} i t_{j}\right)^{-v_{j} / 2} d y .
\end{aligned}
$$

The last one-dimensional integration is equal to the CF of the multivariate $F$ distribution in [24], who obtained it via contour integration. Some other forms of CF including the $p$-dimensional integral form are also available; we refer to [24] for more information. Here, we developed the CF in an easy manner using the stochastic representation given in (5) not depending on contour integration.

When $p=1$, the CF of the (univariate) $F$ distribution becomes

$$
\psi_{X}(t)=\frac{1}{\Gamma\left(v_{0} / 2\right)} \int_{0}^{\infty} e^{-y} y^{\frac{v_{0}+v_{1}}{2}-1}\left(y-\frac{v_{0}}{v_{1}} i t\right)^{-v_{1} / 2} d y .
$$

Let $z=-\frac{v_{0}}{v_{1}} i t$, we find that, by the transformation $x=y / z$,

$$
\begin{aligned}
\psi_{X}(t) & =\frac{z^{v_{0} / 2}}{\Gamma\left(v_{0} / 2\right)} \int_{0}^{\infty} e^{-x z} x^{\frac{v_{0}+v_{1}}{2}-1}(1+x)^{-v_{1} / 2} d x \\
& =\frac{z^{v_{0} / 2}}{\Gamma\left(v_{0} / 2\right)} \Gamma\left(\frac{v_{0}+v_{1}}{2}\right) U\left(\frac{v_{0}+v_{1}}{2}, 1+\frac{v_{0}}{2}, z\right),
\end{aligned}
$$

by (A8) in Appendix A. Furthermore, using (A9) in Appendix A, that is,

$$
U\left(\frac{v_{1}}{2}, 1-\frac{v_{0}}{2}, z\right)=z^{v_{0} / 2} U\left(\frac{v_{0}+v_{1}}{2}, 1+\frac{v_{0}}{2}, z\right),
$$

we have the result (1) after replacing $v_{0}$ with $v_{2}$.

In deriving the $\mathrm{CF}$ of the multivariate $F$ distribution, we found a much shorter derivation of the $\mathrm{CF}$ of the (univariate) $F$ distribution as a by-product compared to the ODE approach. However, to the best of our knowledge, the ODE approach is new in this area, so it is still useful in devising proof for deriving the CF 
of symmetric (Student's $t$-distribution in [22]) and asymmetric distributions such as $F$ and skew-normal distributions. This method could be used to obtain CFs for many other continuous random variables, which is an ongoing project.

\section{Conclusions}

For two asymmetric distributions, $F$ and skew-normal, the CFs are derived by the ODEs, which avoid the computation of the contour integration. The main scenario is to first find an ODE with polynomial coefficients by applying derivatives to the PDF, and then use integration by parts to obtain an ODE satisfied by the CF. Fortunately, the ODEs of the former two distributions are tractable to deduce a formula for the CF. In the $F$ distribution, the calculation process explains why the CF consists of Tricomi's function. The Faddeeva function is applied in the computation of the $\mathrm{CF}$ for the skew-normal distribution. Although this study focuses on the canonical univariate situation, the result can be easily generalized to the case having location and scale parameters and to the multivariate case using simple stochastic representations. The derivation of the $\mathrm{CF}$ for the multivariate $F$ distribution using a stochastic representation herein is much simpler and easier to understand. When the dimensions are limited to one in the multivariate $F$ distribution, the consistency of this $\mathrm{CF}$ with that of the former (univariate) $F$ distribution further suggests that the derivation is reasonable.

Author Contributions: Conceptualization, H.-M.K.; methodology, J.Z.; validation, S.-B.K. and S.-J.K.; formal analysis, J.Z.; investigation, J.Z., S.-B.K. and S.-J.K.; resources, H.-M.K.; writing-original draft preparation, J.Z.; writing-review and editing, S.-B.K., S.-J.K. and H.-M.K.; supervision, H.-M.K.; funding acquisition, H.-M.K. All authors have read and agreed to the published version of the manuscript.

Funding: This research received no external funding.

Acknowledgments: The corresponding author's research was supported by Basic Science Research Program through the National Research Foundation of Korea (NRF) funded by the Ministry of Education (2018R1D1A1B07045603).

Conflicts of Interest: The authors declare no conflict of interest.

\section{Appendix A}

See $[7,26]$ for the following facts. The complete solution of Kummer's equation

$$
z \frac{d^{2} w}{d z^{2}}+(b-z) \frac{d w}{d z}-a w=0
$$

is given by $w(z)=C_{1} M(a, b, z)+C_{2} U(a, b, z)$ where $C_{1}$ and $C_{2}$ are arbitrary constants except that $M(a, b, z)$ does not exist when $b$ is a nonpositive integer. Tricomi's function, $U(a, b, z)$, has the following relationship with Kummer's function ([27]) of the first kind $M$ :

$$
U(a, b, z)=\frac{\Gamma(1-b)}{\Gamma(a-b+1)} M(a, b, z)+\frac{\Gamma(b-1)}{\Gamma(a)} z^{1-b} M(a-b+1,2-b, z),
$$

where

$$
M(a, b, z)=\sum_{s=0}^{\infty} \frac{(a)_{s}}{(b)_{s} s !} z^{s},(a)_{s}=a(a+1) \ldots(a+s-1), \text { and }(a)_{0}=1
$$

Tricomi's function, $U(a, b, z)$, has limiting forms such as

$$
\begin{gathered}
U(a, b, z)=\frac{\Gamma(1-b)}{\Gamma(a-b+1)}+O(z), \Re(b) \leq 0, b \neq 0 \text { as } z \rightarrow 0, \\
U(a, b, z) \sim z^{-a} \text { as } z \rightarrow \infty,
\end{gathered}
$$


where $|\operatorname{ph}(z)|<\frac{3}{2} \pi-\delta$ with an arbitrary small positive constant $\left.\delta \cdot \operatorname{ph}(z)\right)$ is the phase or argument of the complex number $z$, and $\Re(b)$ is the real part of the complex number $b$. Two limiting forms of $M(a, b, z)$ are

$$
\begin{aligned}
M(a, b, z) & =1+O(z) \text { as } z \rightarrow 0 \\
M(a, b, z) & \sim \frac{\Gamma(b)}{\Gamma(a)} e^{z} z^{a-b} \text { as } z \rightarrow \infty
\end{aligned}
$$

where $|\operatorname{ph}(z)|<\frac{1}{2} \pi-\delta$ with an arbitrary small positive constant $\delta$, and $a \neq 0,-1, \ldots$ (polynomial cases). Tricomi's function, $U(a, b, z)$, has the following properties:

$$
\begin{gathered}
\frac{d^{n}}{d z^{n}} U(a, b, z)=(-1)^{n}(a)_{n} U(a+n, b+n, z), \\
(b-a) U(a, b, z)=-U(a-1, b, z)+z U(a, b+1, z), \\
\Gamma(a) U(a, b, z)=\int_{0}^{\infty} e^{-z t} t^{a-1}(1+t)^{b-a-1} d t, \\
U(a, 1-n, z)=z^{n} U(a+n, 1+n, z) .
\end{gathered}
$$

\section{Appendix B}

Lemma A1. The Faddeeva (or Faddeyeva) function ([26] ( Chapter 7) and [28]),

$$
w(z)=e^{-z^{2}}\left(1+\frac{2 i}{\sqrt{\pi}} \int_{0}^{z} e^{u^{2}} d u\right)
$$

is the particular solution of the differential equation

$$
\frac{d w}{d z}+2 z w-\frac{2}{\sqrt{\pi}} i=0
$$

This equation is referred as a differential equation of the Faddeeva function.

Proof of Lemma 1. Derive the first derivative of $w$ at $z$, then the differential equation is obtained.

\section{References}

1. Bisgaard,T.M.; Sasvári, Z. Characteristic Functions and Moment Sequences; Nova Science Publishers, Inc.: New York, NY, USA, 2000.

2. Lukacs, E. Developments in Characteristic Function Theory; Macmillan Publishing Co., Inc.: New York, NY, USA, 1983.

3. Fisher, R.A. On a distribution yielding the error functions of several well-known statistics. Proc. Int. Congr. Math. 1924, 2, 805-813.

4. Ifram, A.F. On the characteristic function of the $F$ - and $t$-distributions. Sankhya Ser. A 1970, 32, 350-352.

5. Johnson, N.L.; Kotz, S. Continuous Univariate Distributions, 2; Houghton-Mifflin: Boston, MA, USA, 1970; p. 78.

6. Awad, A.M. Remark on the characteristic function of the F distribution. Sankhya Ser. A 1980, 42, 128-129.

7. Abramowitz, M.; Stegun, I.A. Handbook of Mathematical Functions with Formulas, Graphs, and Mathematical Tables; Applied Mathematics Series; National Bureau of Standards: Washington, DC, USA, 1983; Chapter 26.

8. Phillips, P.C.B. The true characteristic function of the $F$ distribution. Biometrika 1982, 69, 261-264. [CrossRef]

9. Tricomi, F.G. Funzioni Ipergeometriche Confluenti; Edizioni Cremonese: Roma, Italy, 1954.

10. Witkovsky, V. On the exact computation of the density and of the quantiles of linear combinations of $t$ and $F$ random variables. J. Stat. Plan. Inference 2010, 94, 1-13. [CrossRef]

11. Hwang, S.; Satchell, S.E. Some exact results for an asset pricing test based on the average $F$ distribution. Theor. Econ. Lett. 2012, 2, 435-437. [CrossRef] 
12. Xia, S.; Xiong, Z.; Luo, Y.; Wei, X.; Zhang, G. Effectiveness of the Euclidean distance in high dimensional spaces. Optik 2015, 126, 5614-5619. [CrossRef]

13. O'Hagan, A.; Leonard, T. Bayes estimation subject to uncertainty about parameter constraints. Biometrika 1976, 63, 201-203. [CrossRef]

14. Cook, R.D.; Weisberg, S. An Introduction to Regression Graphics; John Wiley \& Sons: New York, NY, USA, 1994.

15. Azzilini, A.; Capitanio, A. The Skew-Normal and Related Families; Cambridge University Press: New York, NY, USA, 2014.

16. Forina, M.; Armanino, C.; Castino, M.; Ubigli, M. Multivariatedata analysis as a discriminating method of the origin of wines. Vitis $\mathbf{1 9 8 6} 25,189-201$.

17. Pewsey, A. The wrapped skew-normal distribution on the circle. Commun. Stat. 2000, 29, 2459-2472. [CrossRef]

18. Gupta, A.K.; Nguyen, T.T.; Sanqui, J.A.T. Characterization of the skew-normal distribution. Ann. Inst. Stat. Math. 2004, 56, 351-360. [CrossRef]

19. Kim, H.M.; Genton, M.G. Characteristic functions of scale mixtures of multivariate skew-normal distributions. J. Multivar. Anal. 2011, 102, 1105-1117. [CrossRef]

20. Potgieter, C.J.; Genton, M.G. Characteristic function-based semiparametric inference for skew-symmetric models. Scand. J. Stat. 2012, 40, 471-490. [CrossRef]

21. Jiménez-Gamero, M.D.; Kim, H.M. Fast goodness-of-fit tests based on the characteristic function. Comput. Stat. Data Anal. 2015, 89, 172-191. [CrossRef]

22. Gaunt, R.E. A simple proof of the characteristic function of Student's $t$-distribution. Commun. Stat. 2019. [CrossRef]

23. Johnson, N.L.; Kotz, S. Continuous Multivariate Distributions; John Wiley: New York, NY, USA, 1972.

24. Phillips, P.C.B. The Characteristic Function of the Dirichlet and Multivariate F Distributions. In Cowles Foundation for Research in Economics; Cowles Foundation Discussion Papers 865; Yale University: New Haven, CT, USA, 1988.

25. Azzalini, A.; Dalla Valle, A. The multivariate skew-normal distribution. Biometrika 1996, 83, 715-726. [CrossRef]

26. Olver, F.W.J.; Lozier, D.W.; Boisvert, R.F.; Clark, C.W. NIST Handbook of Mathematical Functions; Cambridge University Press: New York, NY, USA, 2010.

27. Kummer, E.E. Über die hypergeometrische Reihe $F(a ; b ; x)$. J. Die Reine Angew. Math. 1836, 15, 39-83.

28. Faddeeva V.N.; Terent'ev, N.M. Tables of Values of the Function $w(z)=e^{-z^{2}}\left(1+2 i \pi^{-1 / 2} \int_{0}^{z} e^{t^{2}} d t\right)$ for Complex Argument; Fok, V.A., Ed.; translated from the Russian by Fry. D. G.; Mathematical Tables Series; Pergamon Press: Oxford, UK, 1961; Volume 11.

Publisher's Note: MDPI stays neutral with regard to jurisdictional claims in published maps and institutional affiliations.

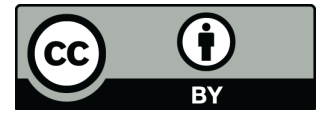

(C) 2020 by the authors. Licensee MDPI, Basel, Switzerland. This article is an open access article distributed under the terms and conditions of the Creative Commons Attribution (CC BY) license (http://creativecommons.org/licenses/by/4.0/). 